las plantas y la amplificación por PCR de un fragmento del gen cfp, permitió detectar el hongo en etapas tempranas de la infección por Cercospora.

Se concluye que los resultados obtenidos constituyen un aporte importante al conocimiento y a la detección temprana de dos de los hongos fitopatógenos más frecuentes que afectan uno de los cultivos más importantes para Argentina y, en particular, para la provincia de Santa Fe.

\section{Phenotypic and genotypic variability in}

Cercospora kikuchii in soybeans culture. early detection of $\mathrm{C}$. kikuchii infection

\section{Summary}

In Santa Fe province, soybean represents one of the greatest sources of revenue. Among the infectious disease-causing agents, fungi of the genus Cercospora have become a significant problem.

This Thesis attempts to contribute to the knowledge of Cercospora species which produced pathology in soybean plants grown in different regions of Santa Fe province during 2005/2006, 2006/2007, 2007/2008 and 2008/2009 campaigns.

The objectives of this work were the isolation and phenotypical characte- rization of the species, followed by a molecular characterization by means of ISSR ("Inter Simple Sequence Repeats"), RAPD-PCR ("Random Amplified Polymorphic DNA"), and PCR amplification of intergenic regions (ITS -"Internally Transcribed Spacers") of DNAr, which were analyzed by RFLP-PCR ("Restriction Fragment-Length Polymorphisms") and finally sequenced.

Petioles and leaflets of plants with visible signs and symptoms of leaf blight and frogeye leaf spot (MOR) were processed.

The Argentinian populations of C. kikuchii and C. sojina are phenotypically, genotypically and geographically variable, thus constituting a very heterogeneous group.

The high degree of similarity between the corresponding ITS fragments and the RFLP patterns of C. kikuchii and C. sojina isolates indicate a high homology in the ITS sequence under study.

Sequentiation results, only allowed confirming that the analyzed fungi belonged to the genus Cercospora, no differences being found between the two species under study.

The last of the objectives proposed was developing early detection methods of Cercospora species in soybean plants.

The Dot Blot and PCR techniques allowed to detect the fungus at the early stage of Cercospora infection.

\title{
Factores que modulan la acción neurotrófica de los estrógenos en el hipocampo del adulto in vivo
}

\author{
Guillermo Samuel Moreno-Piovano \\ gmoreno@fbcb.unl.edu.ar \\ Jorge Guillermo Ramos / Jorgelina Varayoud \\ Laboratorio de Endocrinología y Tumores
}

Hormonodependientes (LETH)

Facultad de Bioquímica y Ciencias Biológicas

Universidad Nacional del Litoral

Fecha de la defensa: 24/07/2013 


\section{Resumen}

El objetivo general de este trabajo fue conocer, mediante el uso de modelos animales in vivo, los mecanismos moleculares por los que ciertas variables neuroendocrino-conductuales pueden modular la acción neurotrófica de los estrógenos en estructuras del cerebro relacionadas con la memoria (hipocampo y sus relaciones corticales y septales). Para esto nos propusimos evaluar en una primera etapa la influencia de un tratamiento agudo con $17 \beta$-estradiol (E2) sobre la regulación transcripcional y postranscripcional de la expresión del $E R \alpha$ en el hipocampo de ratones hembra ovariectomizadas (OVX). Luego de conocer este aspecto básico de regulación pasamos a la segunda etapa (variable tiempo) donde investigamos si el tiempo que el ratón hembra pasa privado de su función ovárica modifica la capacidad transcripcional de la vía estrogénica en el hipocampo. Finalmente en una tercera etapa evaluamos si la exposición de ratones hembra a un ambiente enriquecido modifica los efectos del envejecimiento natural sobre la expresión del ER $\alpha$.

\section{Resultados y Conclusiones}

1) Un tratamiento agudo con E2 tiene la capacidad de modificar la expresión del ER $\alpha$ en el hipocampo del ratón adulto, presentando las siguientes características:

- El tratamiento disminuyó los niveles del ARNm del ER $\alpha$ a las 4 hs. postratamiento. Además, detectamos una menor expresión de la proteína ER $\alpha$ en la región CA1 y el GD del hipocampo.

- La disminución en la transcripción del ER $\alpha$ se produjo en asociación a una menor actividad de los promotores $\mathrm{A}$ y $\mathrm{C}$ del gen.

- El tratamiento con E2 estimuló la expre- sión del ARNm del BDNF pero no de la proteína a las 2 hs. postratamiento. A las 4 hs. postratamiento, no detectamos inducción de la expresión del ARNm de BDNF.

2) El tiempo que un individuo transcurre privado de su función ovárica condiciona el efecto neurotrófico de una terapia hormonal de reemplazo (THR) basada en estrógenos exógenos, pudiéndose destacar las siguientes características:

- El tratamiento con E2 provocó un aumento en la expresión del ARNm y la proteína de BDNF en el hipocampo de los animales que transcurrieron por un período corto de castración (CPE). Si la castración es crónica, la THR basada en estradiol falla en estimular la expresión de BDNF. El aumento en la expresión del ARNm de BDNF observado en el grupo CPE se debió mayormente a la inducción de las variantes EIV-BDNF y EV-BDNF y en menor medida a la variante EII-BDNF.

- El análisis del promotor IV por COBRA reveló que la región regulatoria CRE mostró un mayor número de alelos metilados en los animales que transcurrieron un largo período de castración.

- Al analizar la isla CpG del promotor V se encontró que los grupos experimentales que pasaron mucho tiempo castrados (grupos LPC y LPE) presentaron un mayor grado de metilación respecto a los grupos CP. Estos resultados en paralelo con los obtenidos por COBRA indicarían que la falla en la respuesta estrogénica de BDNF en los animales castrados crónicos está asociada a un silenciamiento transcripcional de los promotores IV y $\mathrm{V}$ del gen de BDNF mediado por un mecanismo de metilación diferencial.

- Los animales que pasaron un corto periodo de castración (CPE) al momento de 
recibir E2 mostraron un incremento significativo de la expresión de SYN en la región CA3. En contraste, el tratamiento con E2 no indujo la expresión de SYN cuando el tratamiento fue iniciado 7,5 meses post-OVX.

3) La expresión del ER $\alpha$ disminuye con la edad avanzada pero esto se revierte por la exposición a un ambiente enriquecido de largo plazo (9 meses):

- La expresión del ARNm total del ER $\alpha$ fue menor en los animales de mediana edad (11 meses). Esta regulación hacia abajo estuvo asociada con una disminución de la actividad relativa de los promotores $A$, C y F del ER $\alpha$.

- La exposición a un ambiente enriquecido no logró revertir la disminución en la transcripción y el incremento en los niveles de metilación del gen de ER $\alpha$ asociados al envejecimiento.

- Sorpresivamente la disminución de la expresión a nivel proteína de ER $\alpha$ que se produce por la edad, fue revertida por la exposición a un ambiente enriquecido de largo plazo.

\section{Factors that modulate the neurotrophic action of estrogen in the hippocampus of adult in vivo}

\section{Summary}

The overall aim of this study was to determine, by using in vivo animal models, the molecular mechanisms by which certain neuroendocrine-behavioral variables can modulate the neurotrophic action of estrogens on brain structures involved in memory (hippocampus and cortical and septal relationships). In a first stage, we decided to assess the influence of an acute treatment with $17 \beta$-estradiol (E2) on transcriptional and post-transcriptional regulation of ER $\alpha$ expression in the hippocampus of ovariectomized female mice. After learning this basic aspect of $\mathrm{ER} \alpha$ regulation we continued with the second stage where we investigated whether the timeframe that the female mouse was deprived of ovarian function modifies the transcriptional capacity of the estrogenic pathway in the hippocampus. Finally, in a third stage we evaluated whether exposure of the female mice to an enriched environment modifies the effects of natural aging on the expression of ER $\alpha$. We found that the period of time that an individual elapses deprived of the ovarian function determines the neurotrophic effect of exogenous E2-based hormone replacement therapy (HRT) by increased methylation of genes encoding neurotrophic factors in the hippocampus. Furthermore, ER $\alpha$ expression in mouse hippocampus decreases with advancing age but this was reversed by exposure to a long term enriched environment. This could favor treatment with HRT to protect cognitive function. 